University of New Orleans

ScholarWorks@UNO

7-1986

\title{
Relationship between the $p$ and s Fresnel reflection coefficients of an interface independent of angle of incidence
}

R. M.A. Azzam

University of New Orleans, razzam@uno.edu

Follow this and additional works at: https://scholarworks.uno.edu/ee_facpubs

Part of the Electrical and Electronics Commons, and the Optics Commons

\section{Recommended Citation}

R. M. A. Azzam, "Relationship between the $p$ and s Fresnel reflection coefficients of an interface independent of angle of incidence," J. Opt. Soc. Am. A 3, 928-929 (1986)

This Article is brought to you for free and open access by the Department of Electrical Engineering at ScholarWorks@UNO. It has been accepted for inclusion in Electrical Engineering Faculty Publications by an authorized administrator of ScholarWorks@UNO. For more information, please contact scholarworks@uno.edu. 


\title{
JOSA COMMUNICATIONS
}

Communications are short articles. Appropriate material for this section includes reports of incidental research results, comments on papers previously published, and short descriptions of theoretical and experimental techniques. Communications are handled much the same as regular articles. Galleys proofs are provided.

\section{Relationship between the $p$ and $s$ Fresnel reflection coefficients of an interface independent of angle of incidence}

\author{
R. M. A. Azzam \\ Department of Electrical Engineering, University of New Orleans, Lakefront, New Orleans, Louisiana 70148
}

Received November 11, 1985; accepted March 8, 1986

\begin{abstract}
The Fresnel reflection coefficients $r_{p}$ and $r_{s}$ of $p$ - and $s$-polarized light at the planar interface between two linear isotropic media are found to be interrelated by $\left(r_{s}-r_{p}\right) /\left(1-r_{s} r_{p}\right)=\cos 2 \beta$, independent of the angle of incidence $\phi$, where $\tan ^{2} \beta=\epsilon$ and $\epsilon$ is the (generally complex) ratio of dielectric constants of the media of refraction and incidence. This complements another relation (found earlier), $\left(r_{s}{ }^{2}-r_{p}\right) /\left(r_{s}-r_{s} r_{p}\right)=\cos 2 \phi$, which is valid at a given $\phi$ independent of $\epsilon$ (i.e., for all possible interfaces). Taken together, these two equations specify $r_{p}$ and $r_{s}$ completely and can be used to replace the original Fresnel equations.
\end{abstract}

The complex-amplitude Fresnel reflection coefficients of $p$ and $s$-polarized monochromatic light at the planar interface between two linear, homogeneous, isotropic, and nonmagnetic media are given by ${ }^{1}$

$$
\begin{aligned}
& r_{p}=\frac{\epsilon \cos \phi-\left(\epsilon-\sin ^{2} \phi\right)^{1 / 2}}{\epsilon \cos \phi+\left(\epsilon-\sin ^{2} \phi\right)^{1 / 2}}=f(\epsilon, \phi), \\
& r_{s}=\frac{\cos \phi-\left(\epsilon-\sin ^{2} \phi\right)^{1 / 2}}{\cos \phi+\left(\epsilon-\sin ^{2} \phi\right)^{1 / 2}}=g(\epsilon, \phi),
\end{aligned}
$$

where $\phi$ is the angle of incidence and $\epsilon$ is the ratio of the dielectric constant of the medium of refraction (which is, in general, complex) to that of the medium of incidence (real).

By eliminating $\epsilon$ between Eqs. (1) and (2), we previously obtained $^{2}$

$$
r_{p}=r_{s}\left(r_{s}-\cos 2 \phi\right) /\left(1-r_{s} \cos 2 \phi\right) .
$$

Equation (3) is a direct relation between $r_{p}$ and $r_{s}$ that is valid for all possible interfaces at a given angle of incidence $\phi$. Its properties and the insight that one can derive from it are discussed in Refs. 2 and 3.

It is apparent that it should also be possible to eliminate $\phi$ between Eqs. (1) and (2) and obtain a second direct relation between $r_{p}$ and $r_{s}$ that is valid for a given interface (or a given $\epsilon)$ at all angles of incidence. After a few algebraic steps, ${ }^{4}$ we get

$$
\left(r_{s}-r_{p}\right) /\left(1-r_{s} r_{p}\right)=c
$$

where

$$
c=(1-\epsilon) /(1+\epsilon) \text {. }
$$

Equation (4) is, to our knowledge, new and can be verified by direct substitution from Eqs. (1) and (2). Equation (4) can be rearranged to read as

$$
r_{p}=\left(r_{s}-c\right) /\left(1-c r_{s}\right)
$$

or

$$
r_{s}=\left(r_{p}+c\right) /\left(1+c r_{p}\right)
$$

each of which is in the form of a bilinear transformation. (For a given complex $\epsilon$, as $\phi$ increases from $0^{\circ}$ to $90^{\circ}, r_{p}$ and $r_{s}$ trace trajectories in the complex plane that are images of each other through such a transformation.)

It is instructive to consider some special cases. At normal incidence $(\phi=0), r_{p}=-r_{s}$ (in the Nebraska conventions ${ }^{5}$ ), and Eq. (4) gives $2 r_{s}=c\left(1+r_{s}^{2}\right)$. When this equation is solved for $r_{s}$, one retrieves the known result $r_{s}(0)=(1-$ $\left.\epsilon^{1 / 2}\right) /\left(1+\epsilon^{1 / 2}\right)$. At grazing incidence, $r_{p}=r_{s}$, and Eq. (4) takes the form $0 /\left(1-r_{s}{ }^{2}\right)=c \neq 0$, from which one correctly obtains $r_{s}^{2}=1$.

At $\phi=45^{\circ}, r_{p}=r_{s}^{2}$; substitution of this result ${ }^{6}$ into Eq. (4) gives $r_{s} /\left(r_{s}^{2}+r_{s}+1\right)=(1-\epsilon) /(1+\epsilon)$. By solving the latter equation for $\epsilon$, one obtains $\epsilon=\left(1+r_{s}^{2}\right) /\left(1+r_{s}\right)^{2}$, a nicelooking result that has proved to be useful recently. ${ }^{7}$

For a dielectric-dielectric interface, $r_{p}=0$ when light is incident at the Brewster angle $\phi=\phi_{B}$. Setting $r_{p}=0$ in Eq. (4) gives

$$
r_{s}\left(\phi_{B}\right)=c=(1-\epsilon) /(1+\epsilon) .
$$

Equation (8) is a simple reduced form of the Fresnel reflection coefficient for the unextinguished $s$ polarization at the Brewster angle. Equation (8) also provides some meaning for the constant $c$. By combining Eqs. (6) and (8), we obtain

$$
r_{p}(\phi)=\frac{r_{s}(\phi)-r_{s}\left(\phi_{B}\right)}{1-r_{s}\left(\phi_{B}\right) r_{s}(\phi)} .
$$

Equation (9) is interesting in that it expresses the reflection coefficient of a dielectric-dielectric interface for the $p$ polarization at any angle $\phi$ in terms of that for the $s$ polarization at the same angle $\phi$ and at the Brewster angle $\phi_{B}$.

For a dielectric-dielectric interface under conditions of total internal reflection (TIR; $\epsilon<1$ and $c>0$ ), we can write

$$
r_{p}=\exp \left(j \delta_{p}\right), \quad r_{s}=\exp \left(j \delta_{s}\right)
$$


where $\delta_{p}$ and $\delta_{s}$ are the phase shifts that the $p$ - and $s$ polarized components of the electric vector experience on TIR. By substituting Eqs. (10) into Eq. (6) and noting that $c$ is real, one gets after some manipulations

$$
\tan \delta_{p}=\frac{\left(1-c^{2}\right) \sin \delta_{s}}{2 c+\left(1+c^{2}\right) \cos \delta_{s}} .
$$

Equation (11) ties directly the TIR phase shifts $\delta_{p}$ and $\delta_{s}$ for a given interface at all angles of incidence, from the critical angle $\phi_{c}=\sin ^{-1} \epsilon^{1 / 2}$ to grazing incidence $\phi=90^{\circ}$.

Before concluding, we note that Eq. (3) can be rewritten as

$$
\left(r_{s}^{2}-r_{p}\right) /\left(r_{s}-r_{s} r_{p}\right)=\cos 2 \phi,
$$

which bears resemblence to Eq. (4). To create more symmetry between the two independent relations between $r_{p}$ and $r_{s}$ at constant $\epsilon$ [Eq. (4)] and at constant $\phi$ [Eq. (12)], we introduce a (generally complex) angle $\beta$ such that

$$
\epsilon=\tan ^{2} \beta \text {. }
$$

(It is interesting to note that $\beta$ reduces to the usual Brewster angle, $\beta=\phi_{B}$, when $\epsilon$ is real, i.e., for an interface between two transparent media.) With Eq. (13), $c$ of Eq. (5) becomes

$$
c=\cos 2 \beta,
$$

and Eq. (4) now reads as

$$
\left(r_{s}-r_{p}\right) /\left(1-r_{s} r_{p}\right)=\cos 2 \beta .
$$

The similarity in structure between Eqs. (12) and (15) is remarkable. ${ }^{8}$ These two equations, taken together, specify $r_{p}$ and $r_{s}$ completely and can be used to replace the original Fresnel equations.

\section{ACKNOWLEDGMENTS}

The kind hospitality of the Département de Physique du Solide, Université de Provence, Marseille, France, where this work was done, a sabbatical from the University of New Orleans, and a Fulbright Senior Research Scholar Award are all gratefully acknowledged.

\section{REFERENCES AND NOTES}

1. See, for example, M. Born and E. Wolf, Principles of Optics (Pergamon, New York, 1975), Sec. 1.5.2.

2. R. M. A. Azzam, "Direct relation between Fresnel's interface reflection coefficients for the parallel and perpendicular polarizations," J. Opt. Soc. Am. 69, 1007-1016 (1979).

3. R. M. A. Azzam, "Relations between amplitude reflectances and phase shifts of the $p$ and $s$ polarizations when electromagnetic radiation strikes interfaces between transparent media," Appl. Opt. 18, 1884-1886 (1979).

4. The simplest way is to form the ratios $U_{\nu}=\left(1-r_{\nu}\right) /\left(1+r_{\nu}\right)$, where $\nu=p, s$. It follows immediately that $U_{s}=\epsilon U_{p}$, from which Eq. (4) is obtained.

5. R. H. Muller, "Definitions and conventions in ellipsometry," Surf. Sci. 16, 14-33 (1969).

6. F. Abelès, "Un théorème rélatif à la réflexion métallique," C. $R$. Acad. Sci. 230, 1942-1943 (1950).

7. R. M. A. Azzam, "Simple and direct determination of complex refractive index and thickness of unsupported or embedded thin films by combined reflection and transmission ellipsometry at $45^{\circ}$ angle of incidence," J. Opt. Soc. Am. 73, 1080-1082 (1983).

8. With $c=\cos 2 \beta$, Eq. (6) also becomes similar to Eq. (3), except for the multiplicative factor $r_{s}$ that appears in the right-hand side of Eq. (3). 